\title{
The complex Burgers equation as a model for collinear interactions of weakly nonlinear shear plane waves in anisotropic elastic materials
}

\author{
Włodzimierz Domański
}

Received: 31 March 2014 / Accepted: 25 June 2014 / Published online: 7 November 2014

(C) The Author(s) 2014. This article is published with open access at Springerlink.com

\begin{abstract}
Collinear interactions of weakly nonlinear quasi-shear plane waves in anisotropic (in particular fiberreinforced) compressible elastic materials are analyzed. Evolution equations for quasi-shear wave amplitudes are derived with the help of the asymptotic method of a double-scale expansion. It is shown that quadratically nonlinear coupling is possible when shear waves propagate along a special fiber direction in anisotropic materials. The evolution equations are reduced to a single inviscid complex Burgers equation when the fiber direction is a threefold symmetry acoustic axis. Some properties of this equation are analyzed. General considerations are illustrated on examples of shear waves propagating along a threefold symmetry acoustic axis in a cubic crystal and in an icosahedral quasicrystal.
\end{abstract}

Keywords Anisotropic elastic materials - Complex Burgers equation · Cubic crystal · Icosahedral quasicrystal · Quadratically nonlinear coupling $\cdot$ Shear waves $\cdot$ Threefold symmetry acoustic axis

\section{Introduction}

We are interested in revealing the manifestation of the anisotropic properties of elastic materials by a collinear propagation of weakly nonlinear quasi-shear plane waves. We analyze the effect of an anisotropy of the elastic material on the form of evolution equations for transverse wave amplitudes. It is known that changes in the amplitudes of weakly nonlinear transverse or quasi-transverse waves in isotropic elastic media are governed by cubically nonlinear evolution equations [1], and quadratic nonlinearity cannot occur in these equations [1]. In the language used in acoustics, this fact is often formulated as follows: second harmonic generation is impossible for shear waves in isotropic materials. However, the presence of fibers in a nonlinear material changes the situation drastically. It turns out that choosing a special direction of transverse wave propagation in an anisotropic material may actually cause the generation of a second harmonic. This manifests itself with the presence of quadratically nonlinear terms in the evolution equations for shear wave amplitudes. Among particular fiber directions we are especially interested in a threefold symmetry acoustic axis. When shear waves propagate along such chosen fiber directions, a quadratically nonlinear coupling occurs in a special way: evolution equations that describe the coupling of a pair of transverse

W. Domański $(\varangle)$

Military University of Technology, Faculty of Cybernetics, Institute of Mathematics and Cryptology,

Gen. S. Kaliskiego 2, 00-908 Warsaw 49, Poland

e-mail: domanski.wlodek@gmail.com 
waves are particularly simple in this case because they contain only one parameter responsible for a nonlinear coupling. Furthermore, coupled equations can be transformed into a single inviscid complex Burgers equation.

Using the expansion of weakly nonlinear geometric optics we have shown [2] what the quadratically nonlinear coupled equations for quasi-shear waves in anisotropic materials look like. Our aim in this work is to derive equations similar to those in [2] but by a different method. Here, unlike the presentation in [2] (see also [3-8]), where the method of weakly nonlinear geometric optics was used, we will apply a double-scale expansion. Instead of introducing a new fast variable and applying geometric optics-type asymptotics, which leads to evolution equations with three independent variables, we introduce here just two new independent variables: a slow time variable $\tau$ and a characteristic variable $\theta$. We concentrate on a special fiber direction of wave propagation, namely, a threefold symmetry acoustic axis.

The paper is organized as follows. After the introduction we present the main equations. Next, in Sect. 3, the double-scale asymptotics is applied and the main evolution equations are derived. The modeling equations for a pair of shear waves propagating along a special fiber direction, which is the threefold symmetry acoustic axis, are then reduced to a single inviscid complex Burgers equation. Some properties of this equation are discussed. In the following section we illustrate our general considerations on examples of a cubic crystal and an icosahedral quasicrystal. The paper ends with concluding remarks.

\section{Main equations}

\subsection{Equations of motion of a continuum}

The equation of motion written in Lagrangian coordinates has the form

$\rho_{0} \frac{\partial \mathbf{v}}{\partial t}=\nabla \cdot \mathbf{T}$

where the first Piola-Kirchhoff stress tensor is

$\mathbf{T}=\mathbf{F} \frac{\partial W}{\partial \mathbf{E}}$,

with the deformation gradient $\mathbf{F}$ and the Green strain $\mathbf{E}$. Here we neglect body forces. The particle velocity is related to the displacement by

$\mathbf{v}=\frac{\partial \mathbf{u}}{\partial t}$

and the deformation gradient is given by the formula

$\mathbf{F}=\mathbf{I}+\nabla \mathbf{u}$

while the Green strain tensor is

$\mathbf{E}=\frac{1}{2}\left(\mathbf{F}^{\mathrm{T}} \mathbf{F}-\mathbf{I}\right)$

We supplement Eq. (1) by the relation between the time derivative of the deformation gradient and the gradient of velocity that follows from (3) and (4):

$$
\frac{\partial \mathbf{F}}{\partial t}=\nabla \mathbf{v}
$$


Therefore, Eqs. (1) and (6) form our system of conservation laws describing the motion of a continuum. We take $\rho_{0}=1$ for simplicity.

\subsection{Constitutive relations}

The elastic material is specified by its strain energy $W$, which is assumed to have the following expansion for small strains:

$W=\frac{1}{2 !} c_{a b c d} E_{a b} E_{c d}+\frac{1}{3 !} c_{a b c d e f} E_{a b} E_{c d} E_{e f}+\cdots$,

where the summation convention on repeated subscripts $a, b, \cdots$ is assumed. The symmetry of the strain implies the relations

$c_{a b c d}=c_{c d a b}=c_{b a c d}, \quad c_{a b c d e f}=c_{c d a b e f}=c_{c d e f a b}=c_{\text {bacdef }}$,

which imply no more than 21 and 56 second- and third-order moduli, respectively.

Equations (2), (4), and (7) together imply that the first Piola-Kirchhoff stress is

$T_{a b}=c_{a b c d} \frac{\partial u_{c}}{\partial x_{d}}+\frac{1}{2} M_{a b c d e f} \frac{\partial u_{c}}{\partial x_{d}} \frac{\partial u_{e}}{\partial x_{f}}+\frac{1}{6} N_{a b c d e f g h} \frac{\partial u_{c}}{\partial x_{d}} \frac{\partial u_{e}}{\partial x_{f}} \frac{\partial u_{g}}{\partial x_{h}}+\cdots$,

where

$M_{a b c d e f}=c_{a b c d e f}+c_{a b d f} \delta_{c e}+c_{b c d f} \delta_{a e}+c_{b d e f} \delta_{a c}$

and

$$
\begin{gathered}
N_{a b c d e f g h}=\frac{1}{2} c_{a b c d e f g h}+c_{c h b c d e f} \delta_{a g}+c_{a b d f g h} \delta_{c e}+\frac{1}{2} c_{a b c d f h} \delta_{e g} \\
+\frac{1}{2} c_{b d e f g h} \delta_{a c}+c_{b d h f} \delta_{c e} \delta_{a g}+\frac{1}{2} c_{b d f h} \delta_{e g} \delta_{a c} .
\end{gathered}
$$

Note that $M_{a b c d e f} \neq M_{\text {bacdef }}$, which implies that the asymmetry of $\mathbf{T}$ is a second-order effect.

\subsection{Plane waves}

For plane waves, $\mathbf{u}$ depends only on one component of $\mathbf{x}$, say $x=\mathbf{x} \cdot \mathbf{n}$, so that

$\nabla \mathbf{u}=\mathbf{m} \otimes \mathbf{n}, \quad$ where $\mathbf{m}=\frac{\partial \mathbf{u}}{\partial x}$.

Thus,

$$
\frac{\partial \mathbf{w}}{\partial t}+\mathbf{A}(\mathbf{w}) \frac{\partial \mathbf{w}}{\partial x}=\mathbf{0},
$$

where

$$
\mathbf{w}=\left(\begin{array}{c}
\mathbf{v} \\
\mathbf{m}
\end{array}\right), \quad \mathbf{A}(\mathbf{w})=-\left(\begin{array}{cc}
\mathbf{0} & \mathbf{B} \\
\mathbf{I} & \mathbf{0}
\end{array}\right) .
$$


The $3 \times 3$ matrix $\mathbf{B}=\mathbf{B}(\mathbf{n})$ is

$\mathbf{B}(\mathbf{n})=\boldsymbol{\Lambda}(\mathbf{n})+\boldsymbol{\Psi}(\mathbf{n}) \mathbf{m}+\frac{1}{2} \boldsymbol{\Pi}(\mathbf{n}) \mathbf{m m}+\cdots$,

where $\boldsymbol{\Lambda}(\mathbf{n}), \boldsymbol{\Psi}(\mathbf{n})$, and $\Pi(\mathbf{n})$ are, in components,

$\Lambda_{a c}(\mathbf{n})=c_{a b c d} n_{b} n_{d}$,

$\Psi_{a c e}(\mathbf{n})=M_{a b c d e f} n_{b} n_{d} n_{f}$,

$\Pi_{\text {aceg }}(\mathbf{n})=N_{a b c d e f g h} n_{b} n_{d} n_{f} n_{h}$,

or, in short,

$B_{a c}=c_{a b c d} n_{b} n_{d}+M_{a b c d e f} n_{b} n_{d} n_{f} m_{e}+\frac{1}{2} N_{a b c d e f g h} n_{b} n_{d} n_{f} n_{h} m_{e} m_{g}+\cdots$.

\subsection{Christoffel tensor}

Matrix A(0) has the following form:

$\mathbf{A}(\mathbf{0})=-\left(\begin{array}{cc}\mathbf{0} & \boldsymbol{\Lambda} \\ \mathbf{I} & \mathbf{0}\end{array}\right)$

The positive-definite property of the second-order moduli implies that $\boldsymbol{\Lambda}$, also known as the Christoffel or acoustical tensor, has in general the following spectral form:

$\mathbf{\Lambda}=\alpha_{1} \mathbf{k}_{1} \otimes \mathbf{k}_{1}+\alpha_{2} \mathbf{k}_{2} \otimes \mathbf{k}_{2}+\alpha_{3} \mathbf{k}_{3} \otimes \mathbf{k}_{3}$,

where $\alpha_{j}>0$, and $\left\{\mathbf{k}_{1}, \mathbf{k}_{2}, \mathbf{k}_{3}\right\}$ is an orthonormal triad of vectors. The six eigenvalues of $\mathbf{A}(\mathbf{0})$ therefore split into three pairs (which may coincide) with opposite signs:

$\lambda_{1}=-\sqrt{\alpha_{1}}=-\lambda_{2}$,

$\lambda_{3}=-\sqrt{\alpha_{2}}=-\lambda_{4}$,

$\lambda_{5}=-\sqrt{\alpha_{3}}=-\lambda_{6}$.

The corresponding right and left eigenvectors of $\mathbf{A}(\mathbf{0})$ are, respectively,

$\mathbf{r}_{2 j-1}=\left(\begin{array}{c}-\lambda_{2 j-1} \mathbf{k}_{j} \\ \mathbf{k}_{j}\end{array}\right), \quad \mathbf{r}_{2 j}=\left(\begin{array}{c}-\lambda_{2 j} \mathbf{k}_{j} \\ \mathbf{k}_{j}\end{array}\right)$

$$
j=1,2,3 \text {. }
$$

$\mathbf{l}_{2 j-1}=\frac{1}{2}\left(\begin{array}{c}-\lambda_{2 j-1}^{-1} \mathbf{k}_{j} \\ \mathbf{k}_{j}\end{array}\right), \quad \mathbf{l}_{2 j}=\frac{1}{2}\left(\begin{array}{c}-\lambda_{2 j}^{-1} \mathbf{k}_{j} \\ \mathbf{k}_{j}\end{array}\right)$.

Note that $\mathbf{l}_{i} \cdot \mathbf{r}_{j}=\delta_{i j}$.

The property $\alpha_{j}>0$ implies that all six eigenvalues $\lambda_{k}, k=1,2, \ldots, 6$ of $\mathbf{A}(\mathbf{0})$ are real. We assume that all the right and left eigenvectors of $\mathbf{A}(\mathbf{0})$ form a full set of linearly independent eigenvectors. The system is therefore hyperbolic at the origin. We are interested in the case where matrix $\mathbf{A}(\mathbf{0})$ has a pair of coinciding eigenvalues. 
Therefore, we assume that there is a fiber direction $\mathbf{n}$ along which a pair of shear waves propagates with a common phase velocity $\lambda$ at the origin. Such a direction is called an acoustic axis; see, for example, [9]. We can then assume that the Christoffel tensor $\boldsymbol{\Lambda}$ from (19) takes the following form [9]:

$\mathbf{\Lambda}=\alpha_{1}\left(\mathbf{I}-\mathbf{k}_{3} \otimes \mathbf{k}_{3}\right)+\alpha_{3} \mathbf{k}_{3} \otimes \mathbf{k}_{3}$,

that is, if $\alpha_{1}=\alpha_{2}$ in (20).

\section{Evolution equations}

\subsection{Asymptotic expansion}

In this section we apply a double-scale expansion method to our problem. Let us consider an initial-value problem (IVP) for a quasilinear hyperbolic system:

$$
\frac{\partial \boldsymbol{w}^{\epsilon}}{\partial t}+\boldsymbol{A}\left(\boldsymbol{w}^{\epsilon}\right) \frac{\partial \boldsymbol{w}^{\epsilon}}{\partial x}=\mathbf{0}, \quad \boldsymbol{w}^{\epsilon}(0, x)=\epsilon \boldsymbol{w}_{1}(0, x)
$$

where $\epsilon$ is a small parameter, $\boldsymbol{w}^{\epsilon}=\left[\boldsymbol{v}^{\epsilon}, \boldsymbol{m}^{\epsilon}\right]^{\mathrm{T}}$, and matrix $\boldsymbol{A}$ is as in (18). We assume that the hyperbolic system (23) is not strictly hyperbolic at the constant state $\mathbf{0}$, and that $\lambda_{1}(\mathbf{0})=\lambda_{2}(\mathbf{0}) \equiv \lambda$ is a double eigenvalue of matrix $\mathbf{A}(\mathbf{0})$ with corresponding left $\mathbf{l}_{k}$ and right $\mathbf{r}_{k}$ eigenvectors, respectively, $k=1,2$, and $\boldsymbol{l}_{\boldsymbol{i}} \cdot \boldsymbol{r}_{\boldsymbol{j}}=\delta_{i j}$, where $\delta_{i j}$ is Kronecker's delta.

We assume a Taylor expansion around the zero constant state:

$\boldsymbol{A}\left(\boldsymbol{w}^{\epsilon}\right)=\boldsymbol{A}(\mathbf{0}+\epsilon \tilde{\boldsymbol{w}})=\boldsymbol{A}(\mathbf{0})+\epsilon \mathcal{B} \tilde{\boldsymbol{w}}+\frac{1}{2} \epsilon^{2} \mathcal{C} \tilde{\boldsymbol{w}} \tilde{\boldsymbol{w}}+\mathcal{O}\left(\epsilon^{3}\right)$

where

$\left.\mathcal{B} \tilde{\boldsymbol{w}} \equiv \nabla_{\boldsymbol{u}}(\boldsymbol{A}(\boldsymbol{w}) \tilde{\boldsymbol{w}})\right|_{\boldsymbol{w}=\mathbf{0}}$,

$\left.\mathcal{C} \tilde{\boldsymbol{w}} \tilde{\boldsymbol{w}} \equiv \nabla_{\boldsymbol{u}}\left(\nabla_{\boldsymbol{u}}(\boldsymbol{A}(\boldsymbol{w}) \tilde{\boldsymbol{w}}) \tilde{\boldsymbol{w}}\right)\right|_{\boldsymbol{w}=\mathbf{0}}$.

We seek a solution to IVP (23) around a $\mathbf{0}$ constant state in the form

$\boldsymbol{w}^{\epsilon}(t, x)=\epsilon \boldsymbol{w}_{1}(\tau, \theta)+\epsilon^{2} \boldsymbol{w}_{2}(\tau, \theta)+\mathcal{O}\left(\epsilon^{3}\right)$.

We introduce two new variables: a slow time $\tau=\epsilon t$ and a characteristic $\theta=x-\lambda t$. We treat them as new independent variables and apply the method of double-scale asymptotics. Let us first calculate derivatives with respect to the old variables $t$ and $x$ and express them in terms of the derivatives with respect to the new variables $\tau$ and $\theta$. We have

$$
\begin{aligned}
\frac{\partial \boldsymbol{w}^{\epsilon}}{\partial t} & =\epsilon \frac{\partial \boldsymbol{w}_{1}}{\partial \tau} \frac{\partial \tau}{\partial t}+\epsilon \frac{\partial \boldsymbol{w}_{1}}{\partial \theta} \frac{\partial \theta}{\partial t}+\epsilon^{2} \frac{\partial \boldsymbol{w}_{2}}{\partial \tau} \frac{\partial \tau}{\partial t}+\epsilon^{2} \frac{\partial \boldsymbol{w}_{2}}{\partial \theta} \frac{\partial \theta}{\partial t}+\mathcal{O}\left(\epsilon^{3}\right) \\
& =\epsilon(-\lambda) \frac{\partial \boldsymbol{w}_{1}}{\partial \theta}+\epsilon^{2}\left(\frac{\partial \boldsymbol{w}_{1}}{\partial \tau}-\lambda \frac{\partial \boldsymbol{w}_{2}}{\partial \theta}\right)+\mathcal{O}\left(\epsilon^{3}\right), \\
\frac{\partial \boldsymbol{w}^{\epsilon}}{\partial x} & =\epsilon \frac{\partial \boldsymbol{w}_{1}}{\partial \theta} \frac{\partial \theta}{\partial x}+\epsilon^{2} \frac{\partial \boldsymbol{w}_{2}}{\partial \theta} \frac{\partial \theta}{\partial x}+\mathcal{O}\left(\epsilon^{3}\right)=\epsilon \frac{\partial \boldsymbol{w}_{1}}{\partial \theta}+\epsilon^{2} \frac{\partial \boldsymbol{w}_{2}}{\partial \theta}+\mathcal{O}\left(\epsilon^{3}\right) .
\end{aligned}
$$


Taking into account (24), (28), and (29) we obtain

$$
\frac{\partial \boldsymbol{w}^{\epsilon}}{\partial t}+\boldsymbol{A}\left(\boldsymbol{w}^{\epsilon}\right) \frac{\partial \boldsymbol{w}^{\epsilon}}{\partial x}=\epsilon(\boldsymbol{A}(\mathbf{0})-\lambda \boldsymbol{I}) \frac{\partial \boldsymbol{w}_{1}}{\partial \theta}+\epsilon^{2}\left(\frac{\partial \boldsymbol{w}_{1}}{\partial \tau}+\mathcal{B} \boldsymbol{w}_{1} \frac{\partial \boldsymbol{w}_{1}}{\partial \theta}+(\boldsymbol{A}(\mathbf{0})-\lambda \boldsymbol{I}) \frac{\partial \boldsymbol{w}_{2}}{\partial \theta}\right)+\mathcal{O}\left(\epsilon^{3}\right) .
$$

Now, gathering together terms that appear with like powers of a small parameter $\epsilon$, we equate these consecutive terms to zero and obtain the following equations:

- $\mathcal{O}(\epsilon)$ terms vanish $\Leftrightarrow\left(\boldsymbol{A}(\mathbf{0})-\lambda_{j} \boldsymbol{I}\right) \frac{\partial \boldsymbol{w}_{1}}{\partial \theta}=\mathbf{0}$.

Since we focus on shear waves, we take a solution of these equations in the form

$\boldsymbol{w}_{1}(\tau, \theta)=a_{1}(\tau, \theta) \boldsymbol{r}_{1}+a_{2}(\tau, \theta) \boldsymbol{r}_{2}$

with $a_{j}(\tau, \theta)$ the unknown amplitudes of quasi-shear waves and $\boldsymbol{r}_{j}$ the corresponding eigenvectors of matrix $\boldsymbol{A}(\mathbf{0})$;

- $\mathcal{O}\left(\epsilon^{2}\right)$ terms vanish $\Leftrightarrow(\boldsymbol{A}(\mathbf{0})-\lambda \boldsymbol{I}) \frac{\partial \boldsymbol{w}_{2}}{\partial \theta}=-\mathcal{F}$

with

$\mathcal{F} \equiv \mathcal{B} \boldsymbol{w}_{1} \frac{\partial \boldsymbol{w}_{1}}{\partial \theta}+\frac{\partial \boldsymbol{w}_{1}}{\partial \tau}$

The inhomogeneous algebraic equation (33) has a nonzero solution provided the right-hand side of (33) is orthogonal to the left eigenvectors of matrix $\boldsymbol{A}(\mathbf{0})$. Let us take two such eigenvectors $\boldsymbol{l}_{1}$ and $\boldsymbol{l}_{2}$, which correspond to a pair of shear waves propagating in the reference state with a common phase speed $\lambda$. From the solvability condition we have in particular

$\boldsymbol{l}_{1} \cdot \mathcal{F}=0$,

$\boldsymbol{l}_{2} \cdot \mathcal{F}=0$.

Let us introduce the following notation:

$\Gamma_{p q}^{j}=\boldsymbol{l}_{j} \cdot\left(\left.\nabla_{\boldsymbol{w}} \boldsymbol{A}(\boldsymbol{w})\right|_{\boldsymbol{w}=\mathbf{0}}\right) \boldsymbol{r}_{p} \boldsymbol{r}_{q}$

for the interaction coefficient. Taking into account (25), (32), and (34) we can write conditions (35) and (36) as follows:

$\frac{\partial a_{1}}{\partial \tau}+\Gamma_{11}^{1} a_{1} \frac{\partial a_{1}}{\partial \theta}+\Gamma_{21}^{1} a_{2} \frac{\partial a_{1}}{\partial \theta}+\Gamma_{12}^{1} a_{1} \frac{\partial a_{2}}{\partial \theta}+\Gamma_{22}^{1} a_{2} \frac{\partial a_{2}}{\partial \theta}=0$,

$\frac{\partial a_{2}}{\partial \tau}+\Gamma_{11}^{2} a_{1} \frac{\partial a_{1}}{\partial \theta}+\Gamma_{21}^{2} a_{2} \frac{\partial a_{1}}{\partial \theta}+\Gamma_{12}^{2} a_{1} \frac{\partial a_{2}}{\partial \theta}+\Gamma_{22}^{2} a_{2} \frac{\partial a_{2}}{\partial \theta}=0$.

We obtain the pair of coupled nonlinear partial differential equations that are the asymptotic evolution equations for the amplitudes of the pair of quasi-shear elastic waves in the vicinity of a double umbilic point.

\subsection{Interaction coefficients}

The interaction coefficients can be represented as

$\Gamma_{p, q}^{j}=\frac{1}{2} \lambda_{j}^{-1} \mathbf{k}_{\left\lfloor\frac{j+1}{2}\right\rfloor} \cdot \boldsymbol{\Psi} \mathbf{k}_{\left\lfloor\frac{p+1}{2}\right\rfloor} \mathbf{k}_{\left\lfloor\frac{q+1}{2}\right\rfloor}$, 
where $L\rfloor$ denotes the floor function, that is, the largest integer less than or equal to a given number. Using Cartesian components with the notation $\mathbf{k}_{j} \equiv k_{1}^{j} \mathbf{e}_{1}+k_{2}^{j} \mathbf{e}_{2}+k_{3}^{j} \mathbf{e}_{3}$, we can express further the interaction coefficients as follows:

$$
\begin{aligned}
\Gamma_{p, q}^{j}= & \frac{1}{2} \lambda_{j}^{-1}\left[c_{a b c d e f} n_{b} n_{d} n_{f} k_{a}^{\lfloor(j+1) / 2\rfloor} k_{c}^{\lfloor(p+1) / 2\rfloor} k_{e}^{\lfloor(q+1) / 2\rfloor}\right. \\
& \left.+c_{a b c d} n_{b} n_{c} n_{d}\left(k_{a}^{\lfloor(j+1) / 2\rfloor} \delta_{p q}+k_{a}^{\lfloor(p+1) / 2\rfloor} \delta_{j q}+k_{a}^{\lfloor(q+1) / 2\rfloor} \delta_{j p}\right)\right] \\
= & \frac{1}{2} \lambda_{j}{ }^{-1} c_{a b c d e f} n_{b} n_{d} n_{f} k_{a}^{\lfloor(j+1) / 2\rfloor} k_{c}^{\lfloor(p+1) / 2\rfloor} k_{e}^{\lfloor(q+1) / 2\rfloor} \\
& +\frac{1}{2} \lambda_{j} \mathbf{n} \cdot\left(\mathbf{k}_{\lfloor(j+1) / 2\rfloor} \delta_{p q}+\mathbf{k}_{\lfloor(p+1) / 2\rfloor} \delta_{j q}+\mathbf{k}_{\lfloor(q+1) / 2\rfloor} \delta_{j p}\right) .
\end{aligned}
$$

The assumption of hyperelasticity (2) implies that interaction coefficients (37) have the following symmetry property:

$\Gamma_{p, q}^{j}=\Gamma_{q, p}^{j}$.

It follows from the preceding formulas that the coefficients have in our case, in addition to the general property (42), the following indicial symmetries:

$\Gamma_{p, q}^{j}=\Gamma_{j, q}^{p}$.

This makes them totally symmetric under the interchange of indices. In particular, note that

$\Gamma_{2,2}^{1}=\Gamma_{1,2}^{2} \equiv \Gamma_{2}^{1}$,

$\Gamma_{1,1}^{2}=\Gamma_{1,2}^{1} \equiv \Gamma_{1}^{2}$.

Using the notation $\Gamma^{1} \equiv \Gamma_{1,1}^{1}$ and $\Gamma^{2}=\Gamma_{2,2}^{2}$, and summarizing we have the following lemma.

Lemma 1 The evolution equations for the amplitudes of shear waves propagating along an acoustic axis are

$$
\begin{aligned}
& \frac{\partial a_{1}}{\partial \tau}+\frac{1}{2}\left(\Gamma_{1} \frac{\partial a_{1}^{2}}{\partial \theta}+2 \Gamma_{1}^{2} \frac{\partial\left(a_{1} a_{2}\right)}{\partial \theta}+\Gamma_{2}^{1} \frac{\partial a_{2}^{2}}{\partial \theta}\right)=0, \\
& \frac{\partial a_{2}}{\partial \tau}+\frac{1}{2}\left(\Gamma_{1}^{2} \frac{\partial a_{1}^{2}}{\partial \theta}+2 \Gamma_{2}^{1} \frac{\partial\left(a_{1} a_{2}\right)}{\partial \theta}+\Gamma_{2} \frac{\partial a_{2}^{2}}{\partial \theta}\right)=0 .
\end{aligned}
$$

The nonlinear terms in the equations involve four coefficients: $\Gamma_{1}, \Gamma_{2}, \Gamma_{1}^{2}$, and $\Gamma_{2}^{1}$, the last two of which determine the coupling between the equations.

\subsection{Threefold axis}

Evolution equations simplify even more if we assume that the propagation direction is a threefold symmetry axis. In this case we obtain that $\Gamma_{1}+\Gamma_{2}^{1}=0$ and that $\Gamma_{2}=\Gamma_{1}^{2}=0$ (see [6] or [2] for details). Therefore, we can formulate the following lemma. 
Lemma 2 The evolution equations for the amplitudes of shear waves propagating along an acoustic axis that is a threefold symmetry axis are

$$
\begin{aligned}
\frac{\partial a_{1}}{\partial \tau}+\frac{1}{2} \Gamma_{2}\left(\frac{\partial a_{1}^{2}}{\partial \theta}-\frac{\partial a_{2}^{2}}{\partial \theta}\right) & =0, \\
\frac{\partial a_{2}}{\partial \tau}-\Gamma_{2} \frac{\partial\left(a_{1} a_{2}\right)}{\partial \theta} & =0 .
\end{aligned}
$$

Only one coefficient, $\Gamma_{2}$, determines the coupling between the evolution equations when the propagation direction is a threefold symmetry axis.

In the next lemma we reveal the formula for the coefficient $\Gamma_{2}$.

Lemma 3 The coefficient $\Gamma_{2}$ from (46) can be expressed in the form

$\Gamma_{2}=\lambda_{2}^{-1} G_{2}$

where $G_{2}$ is expressed entirely in terms of third-order elastic constants:

$G_{2}=\frac{1}{2} c_{a b c d e f} n_{b} n_{d} n_{f} k_{a}^{1} k_{c}^{1} k_{e}^{2}$

The proof follows from the explicit formula for the coefficient $\Gamma_{2}$ by a simple inspection. Since $\mathbf{n} \cdot \mathbf{k}_{1}=0$, hence the last term in (41) disappears and we obtain (47) and (48); see [2] for more details.

By introducing a complex variable, system (46) can be transformed into the single complex Burgers equation. We will show how to do this in the next section.

\subsection{The complex Burgers equation}

To simplify the consideration, let us introduce the following notation $a_{1}(\tau, \theta) \equiv u(t, x), a_{2}(\tau, \theta) \equiv v(t, x)$, and let the subscript denote a partial derivative; so for example,

$\frac{\partial a_{1}}{\partial \tau} \equiv u_{t}, \quad \frac{\partial a_{1}}{\partial \theta} \equiv u_{x}$,

and similarly

$\frac{\partial a_{2}}{\partial \tau} \equiv v_{t}, \quad \frac{\partial a_{2}}{\partial \theta} \equiv v_{x}$.

Moreover, assume that the coefficient $\Gamma_{2}$ is normalized to 1.

Under these assumptions, system (46) reduces to

$u_{t}+\frac{1}{2}\left(u^{2}-v^{2}\right)_{x}=0, \quad v_{t}-(u v)_{x}=0$.

Suppose we add the first equation of (49) to the second equation multiplied by the imaginary i. We then obtain

$u_{t}+\mathrm{i} v_{t}+\frac{1}{2}\left(u^{2}-2 \mathrm{i} u v-v^{2}\right)_{x}=0$.

Now, assuming that $U \equiv u+\mathrm{i} v$, we have that the complex conjugate squared $\bar{U}^{2}=u^{2}-2 \mathrm{i} u v-v^{2}$, hence system (49) can be written as a single complex Burgers equation: 
$U_{t}+\frac{1}{2}\left(\bar{U}^{2}\right)_{x}=0$

Similarly, by subtracting the second equation of (49) multiplied by imaginary i from the first one, we obtain the complex conjugate of (51):

$\bar{U}_{t}+\frac{1}{2}\left(U^{2}\right)_{x}=0$.

We can therefore formulate the following lemma.

Lemma 4 By introducing the complex variable $U \equiv u+\mathrm{i} v$, system (49) can be transformed into the complex Burgers Eq. (51) or its complex conjugate (52).

Definition 1 We say that the periodic function $U(t, x)$ with period $L,(U(t, x)=U(t, x+L))$, has a threefold symmetry if for all $t$ and $x$

$U\left(t, x+\frac{L}{3}\right)=\mathrm{e}^{2 \pi \mathrm{i} / 3} U(t, x)$.

Lemma 5 If $U(t, x)$ satisfies the complex Burgers Eq. (51), then so does $V(t, x) \equiv \mathrm{e}^{2 \pi \mathrm{i} / 3} U(t, x)$.

Proof We have $\bar{V}^{2}=\mathrm{e}^{-4 \pi \mathrm{i} / 3} \bar{U}^{2}$, so

$V_{t}+\frac{1}{2}\left(\bar{V}^{2}\right)_{x}=\mathrm{e}^{2 \pi \mathrm{i} / 3} U_{t}+\frac{1}{2} \mathrm{e}^{-4 \pi \mathrm{i} / 3}\left(\bar{U}^{2}\right)_{x}=\mathrm{e}^{2 \pi \mathrm{i} / 3}\left[U_{t}+\frac{1}{2}\left(\bar{U}^{2}\right)_{x}\right]=0$.

From Lemma 5 and Definition 1 it follows that a periodic function with a threefold symmetry satisfies (49). Some solutions to the complex Burgers Eqs. (49) were studied by Noelle in his thesis [10]. These equations reveal interesting features like, for example, the formation of nonclassical-undercompressive shock waves. The problem of stability of such shocks for complex Burgers equations was investigated in [11].

Let us now revisit system (49) and prove the following lemma.

Lemma 6 System (49) is not genuinely nonlinear.

Proof We write system (49) in a quasilinear form:

$\frac{\partial \boldsymbol{W}}{\partial t}+\boldsymbol{C}(\boldsymbol{W}) \frac{\partial \boldsymbol{W}}{\partial x}=\mathbf{0}$,

where $\boldsymbol{W}=[u, v]^{\mathrm{T}}$, and

$\boldsymbol{C}(\boldsymbol{W})=\left(\begin{array}{cc}u & -v \\ -v & -u\end{array}\right)$

The eigenvalues are

$\lambda_{1}(\boldsymbol{W})=-\sqrt{u^{2}+v^{2}}=-|\boldsymbol{W}|, \quad \lambda_{2}(\boldsymbol{W})=\sqrt{u^{2}+v^{2}}=|\boldsymbol{W}|$.

We have

$\nabla_{\boldsymbol{W}} \lambda_{1}(\boldsymbol{W})=-\frac{1}{|\boldsymbol{W}|}(u, v), \quad \nabla_{\boldsymbol{W}} \lambda_{2}(\boldsymbol{W})=\frac{1}{|\boldsymbol{W}|}(u, v)$.

The corresponding eigenvectors have the form

$\boldsymbol{r}_{1}(\boldsymbol{W})=[-u+|\boldsymbol{W}|, v], \quad \boldsymbol{r}_{2}(\boldsymbol{W})=[-u-|\boldsymbol{W}|, v]$. 
The genuine nonlinearity condition means that

$\nabla_{\boldsymbol{W}} \lambda_{j}(\boldsymbol{W}) \cdot \boldsymbol{r}_{j} \neq 0 \quad j=1,2$.

We have

$\nabla_{\boldsymbol{W}} \lambda_{1}(\boldsymbol{W}) \cdot \boldsymbol{r}_{1}=\frac{u^{2}-v^{2}-u|\boldsymbol{W}|}{|\boldsymbol{W}|}, \quad \nabla_{\boldsymbol{W}} \lambda_{2}(\boldsymbol{W}) \cdot \boldsymbol{r}_{2}=\frac{v^{2}-u^{2}-u|\boldsymbol{W}|}{|\boldsymbol{W}|}$.

One can easily check that the genuine nonlinearity is lost if $v^{2}=3 u^{2}$ for $u<0$ in the first family and $u>0$ in the second family.

\section{Applications}

In this section we apply our theory to a cubic crystal and an icosahedral quasicrystal. First, we simplify the notation for elastic constants. We use Brugger's second-order $c_{i j}$ and third-order $c_{i j k}$ elastic constants (see [12]) instead of $c_{a b c d}$ and $c_{a b c d e f}$, respectively, where the change of indices is done according to the following rule:

$$
\begin{array}{lll}
11 \rightarrow 1 & 22 \rightarrow 2 & 33 \rightarrow 3 \\
23 \rightarrow 4 & 32 \rightarrow 4 & 13 \rightarrow 5 \\
31 \rightarrow 5 & 12 \rightarrow 6 & 21 \rightarrow 6
\end{array}
$$

\subsection{Cubic crystal}

There are five cubic crystal classes, denoted by $23, m 3,432, m 3 m$, and $\overline{4} 3 m$. All of these cubic crystals possess trigonal symmetry with respect to the four cube diagonals. All cubic crystals also have a diagonal symmetry about the three cubic axes. Only two of the cubic crystal classes have an additional tetragonal symmetry about the cubic axes. Each symmetry diminishes the number of elastic stiffness constants. The number of constants for the most symmetric of cubic crystals is reduced to three second-order constants and six third-order constants [5]. We will be interested in the most symmetric of cubic crystals - those belonging to the class $m 3 m$. This is motivated by the fact that many crystals appearing in nature belong to this class, for example, all metals of cubic symmetry, $\mathrm{Au}, \mathrm{Fe}, \mathrm{Ni}$, $\mathrm{Ag}$, and ionic crystals such as, for example, $\mathrm{NaCl}, \mathrm{KCl}, \mathrm{LiF}, \mathrm{AgCl}$, and many more.

Let us consider a cubic crystal of class $m 3 m$ in which the strain energy $W$ is defined by three second-order and six third-order elastic constants [5]:

$W=W\left(c_{11}, c_{12}, c_{44}, c_{111}, c_{112}, c_{144}, c_{123}, c_{166}, c_{456}\right)$.

We study the propagation of a pair of shear waves along an axis of threefold symmetry, $\mathbf{n}=\frac{1}{\sqrt{3}}\left[\begin{array}{lll}1 & 1 & 1\end{array}\right]$, which is also an acoustic axis. We discover that shear wave amplitudes are described by coupled evolution Eqs. (46) with the coefficient $\Gamma_{2}$ given by formula (47), with

$\lambda_{2}=\sqrt{\frac{c_{11}-c_{12}+c_{44}}{3}} \quad$ and $\quad G_{2}=\frac{1}{18 \sqrt{2}}\left[c_{111}+2 c_{123}-2 c_{456}-3\left(c_{112}-c_{144}+c_{166}\right)\right]$.

\subsection{Icosahedral quasicrystal}

The discovery of quasicrystals (see [13] and [14]) spurred strong interest in various properties of these strange solids with unusual symmetry axes that are incompatible with periodicity and without translational symmetry in space.

Icosahedral quasicrystals, besides a phonon phase, also have a phason phase, so in general their energy density function is characterized by 5 second-order elastic constants ( 2 due to the phonon field, 2 due to the phason field, 
and 1 associated with phonon-phason coupling) [15] and 20 third-order elastic constants (4 due to phonon fields, 5 due to phason fields, 4 due to phonon-phonon-phason coupling, and 7 due to phonon-phason-phason coupling) [16]. Of these third-order constants only four are independent, and, moreover, the following relations are satisfied (see [17] with corrections from [18]):

$$
\begin{aligned}
& c_{111}=c_{123}+2 c_{144}+2\left(c_{155}+c_{166}\right), \\
& c_{112}=c_{123}+2 c_{144}+\left(2 \cos \frac{\pi}{5}-1\right)\left(c_{155}-c_{166}\right), \\
& c_{113}=c_{123}+2 c_{144}+2 c_{144}+2 \cos \frac{\pi}{5}\left(c_{155}-c_{166}\right), \\
& c_{456}=-\frac{1}{2} c_{144}-\frac{1}{2}\left(2 \cos \frac{\pi}{5}-1\right) c_{155}+\cos \frac{\pi}{5} c_{166} .
\end{aligned}
$$

Following [19] we consider the icosahedral quasicrystal AlPdMn, which is phason-free and is characterized by two second-order and five third-order elastic constants, so it is linearly isotropic but anisotropic in a nonlinear range. The strain energy density function is

$$
W=W\left(c_{11}, c_{44}, c_{111}, c_{112}, c_{113}, c_{456}\right) \text {. }
$$

We investigate the propagation of collinear transverse waves along the fiber direction, which is a threefold symmetry acoustic axis. In this case, the evolution equations for a pair of such waves reduce to system (46), with the coefficient $\Gamma_{2}$ given by formula (47), with $\lambda_{2}=\sqrt{c_{44}}$ and $G_{2}=(4 / 9)\left(c_{112}-c_{113}\right)$.

\section{Concluding remarks}

The possibility of the appearance of quadratically nonlinear coupling in the evolution equations for shear elastic waves propagating along the threefold symmetry acoustic axis direction in a cubic crystal was shown for the first time in our paper [3]; see also [4,5]. Experimental confirmation of this fact was announced in [20]. Similar coupling was detected in an icosahedral quasicrystal $[18,19]$. The problem of the second harmonic generation of shear waves in crystals has been investigated in [21]; see also the recent paper [22].

It seems that a complex Burgers equation appeared for the first time in Lax's paper [23]. The properties of the solution to this equation were studied by Lax's Ph.D. student Sebastian Noelle [10]. This equation appeared for a model of various phenomena, for example, as a one-dimensional model for the dynamics of dislocations in crystals [24] and in studying surfaces in $\mathcal{R}^{3}$ that arise as limit shapes in random surface models related to planar dimers [25].

Open Access This article is distributed under the terms of the Creative Commons Attribution License which permits any use, distribution, and reproduction in any medium, provided the original author(s) and the source are credited.

\section{References}

1. Goldberg Z (1960) On self-interaction of plane longitudinal and transverse waves. Sov Phys Acoust 6:307-310

2. Domański W, Norris AN (2009) Degenerate weakly nonlinear elastic plane waves. Int J Nonlinear Mech 44:486-493

3. Domański W (1999) Asymptotic equations or weakly nonlinear elastic waves in a cubic crystal. In: Fey M, Jeltsch R (eds) Hyperbolic problems: theory, numerics, applications, ISNM, vol 129. Birkhäuser, Basel, pp 232-241

4. Domański W (2000) Finite amplitude elastic waves in a cubic crystal. In Lauterborn W, Kurz T (eds) Nonlinear acoustics at the turn of the millenium: ISNA 15, AIP, pp 249-252

5. Domański W (2000) Weakly nonlinear elastic plane waves in a cubic crystal. Contemp Math 255:45-61

6. Domański W (2006) Propagation and interaction of hyperbolic plane waves in nonlinear elastic solids, IFTR Reports 4, Warsaw, pp $1-169$ 
7. Domański W (2008) Propagation and interaction of weakly nonlinear elastic plane waves in a cubic crystal. Wave Motion 45:337349

8. Domański W, Norris A N (2008) Nonlinear evolution equations for degenerate transverse waves in anisotropic elastic solids. In Enflo B, Hedberg C M, Kari L, (eds) Nonlinear Acoustics-Fundamentals and Applications, 18th International Symposium on Nonlinear Acoustics, AIP Conference Proceedings AIP vol 1022, pp 259-262

9. Norris AN (2004) Acoustic axes in elasticity. Wave Motion 40:315-328

10. Noelle S (1990) Cauchy problems for the complex Burgers equation in one and two space dimensions. Ph D thesis, New York University

11. Liu TP, Zumbrun K (1995) Nonlinear stability of an undercompressive shock for complex Burgers equation. Comm Math Phys 168:163-186

12. Brugger K (1964) Thermodynamic definition of higher order elastic coefficients. Phys Rev 33:1611-1622

13. Shechtman D, Blech I, Gratias D, Cahn JW (1984) Metallic Phase with long-gange orientational order and no translational symmetry. Phys Rev Lett 53:1951-1954

14. Levine D, Steinhardt PJ (1984) Quasicrystals: a new class of ordered structures. Phys Rev Lett 53:2477-2480

15. Levine D, Lubensky TC, Ostlund S, Ramaswamy S, Steinhardt PJ, Toner J (1985) Elasticity and dislocations in pentagonal and icosahedral quasicrystals. Phys Rev Lett 54:1520-1523

16. Ricker M, Trebin HR (2002) Nonlinear generalized elasticity of icosahedral quasicrystals. J Phys A Math Gen 35:6953-6962

17. Chen LC, Ebalard S, Goldman LM, Ohashi W, Park B, Spaepen F (1986) The number of third-order elastic constants of an icosahedral solid. J Appl Phys 60:2638

18. Duquesne JY, Perrin B (2000) Anisotropic nonlinear elastic properties of an icosahedral quasicrystal. Phys Rev Lett 85:4301-4304

19. Duquesne JY, Perrin B (2002) Elastic wave interaction in icosahedral AlPdMn. Physica B 316-317:064303

20. Duquesne JY, Perrin B (2001) Interaction of collinear transverse acoustic waves in cubic crystals. Phys Rev B 63:064303

21. Jiang W, Cao W (2004) Second harmonic generation of shear waves in crystals. IEEE Trans Ultrason Ferroelectr Freq Control 51:153-162

22. Liu X, Jiang W, Cao W, Jiang Z (2014) Interaction of collinear acoustic waves propagating along pure mode directions in crystals. J Appl Phys 115:064909

23. Lax P D (1986) Hyperbolic systems of conservation laws in several space variables. In Ohya Y, Kasahara K, Shimakura N (eds) Current topics in partial differential equations. Tokyo, pp 327-341

24. Biler P, Karch G, Monneau R (2010) Nonlinear diffusion of dislocation density and self-similar solutions. Comm Math Phys 294:145-168

25. Kenyon R, Okounkov A (2007) Limit shapes and the complex Burgers equation. Acta Math 199:263-302 